\section{Consenso Brasileiro de Hipertensão Arterial}

$\mathbf{O}$ III Congresso Brasileiro de Hipertensão Arterial teve como objetivos revisar, atualizar e expandir o documento do II Consenso Brasileiro de Hipertensão Arterial, de 1994, produzindo um novo documento préatico, objetivo $\mathrm{e}$ adequado à realidade brasileira, para ser utilizadocomo referência no diagnóstico e tratamento da hipertensão arterial pela comunidade médica e de profissionais da saúde do país que atendem o hipertenso.

Para isso, sob os auspícios das Sociedades Brasileiras de Hipertnsão, Cardiologia e Nefrologia, reuniram-se, em Campos do Jordão, no período de 12 a 14 de Fevereiro de 1998, 84 especialistas em hiperetensão arterial procedentes de 17 Estados brasileiros. Participaram, também, representantes das Sociedades Brasileiras de Clínica Médica, Endocrinologia, Pediatria, Geriatria e Gerontologia, da Academia Brasileira de Neurologia, da Federação Brasileira de Ginecologia e Obstetrícia e da Divisão de Doenças Cardiovasculares do Ministério da Saúde.

Diagnóstico realizado o mais precocemente possivel e tratamento adequado e contínuo são armas importantes no tratamento da hipertensão arterial. Entretanto, sabe-se que a prevenção do aumento da pressão arterial constitui o meio mais eficiente de combater a hipertensão arterial as dificuldades e o elevado custo social de suas complicações. Assim, a reunião do III Consenso Brasileiro de Hipertensão Arterial, além de revisar e atualizaros conhecimentos para o diagnóstico e tratamento do paciente hipertenso, teve também a preocupação de discutir e propor medidas visando à prevenção da hipertensão arterial e das doenças cardiovasculares a ela relacionadas. Foi criado, para tanto, um capítulo especifico destinado à prevenção primária da hipertensão arterial, onde é enfatizada a necessidade de implementação de medidas desde a infância, visando modificações do estilo de vida e o combate aos fatores de risco cardiovasculares.

Nessa reunião, pela primeira vez, contou-se também com a experiência de profissionais de saúde não médicos com grande vivência na abordagem multiprofissionais do hipertenso, o que permitiu adicionar novo e importante capítulo no documento do Consenso: "A abordagem multiprofissional do hipertenso". Por ter sido essa a primeira vez que a reunião do Consenso contou a participação desses profissionais, serão aqui destacados seus nomes: enfermeiras Angela Pierin, Ana Luisa Sousa, Cátia Palmeira, Fátima Lúcia Machado Braga, Jussara Melo e Nárcia Elisa Bellucci Kohlmann; nutricionistas AnaCarolina Vasconcellos, Estelamaris Tronco Monego e Neide de Jesus; assistente social Denise Mattos Dias, psicóloga Marilda Lippi; e professor de educação física Carlos Edurado Negrão.

A Comissão Organizadora gostaria de agradecer o empenho de todos os colegas que contribuíram de forma definitiva para o sucesso da reunião, e particularmente a Comissão de Redação, pelo excelente trabalho na confecção destc documentto. Faz, ainda, agradecimento especial aos laboratórios Asta Médica, Astra, Bayer, Biosintética, Bristol-Myers Squibb, Glaxo Wellcome, Hoechst Marion Roussel, Merck Sharp \& Dohme, Novartis, Pfizer, Roche, Sanofi Winthrop, Servier e Zeneca, pelo apoio institucional, que permitiram a realização da reunião do III Consenso Brasileiro de Hipertensão Arterial e a ampla divulgação do documento gerado

\section{apresentação}

\author{
Osvaldo Kohlmann Jr. \\ Armênio Costa Guimarães \\ Maria Helena C. Carvalho \\ Hilton de Castro Chaves Jv. \\ Carlos Alberto Machado \\ José Nery Praxedes \\ José Luiz Santello
}

Comissão Organizadora 


\section{Consenso Brasileiro de Hipertensão Arterial}

\section{Promoção}

Sociedade Brasileira de Hipertensão

Sociedade Brasileira de Cardiologia - Departamento de Hipertensão Arterial

Sociedade Brasileira de Nefrologia - Departamento de Hipertensão Arterial

\section{Comissão}

\section{Organizadora}

Osvaldo Kohlmann Jr.

Armênio Costa Guimarães

Maria Helena Catelli Carvalho

Hilton de Castro Chaves Jr.

Carlos Alberto Machado

José Nery Praxedes

José Luiz Santello

\section{Comissão de \\ Redação}

Osvaldo Kohlmann Jr.

Armênio Costa Guimarães

Maria Helena Catelli Carvalho

Hilton de Castro Chaves Jr.

Carlos Alberto Machado

José Nery Praxedes

José Luiz Santello

Fernando Nobre

Décio Mion Jr.

\section{Apoio \\ Institucional}

Asta Medica

Astra Zeneca

Bayer

Biosintética

Bristol-Myers Squibb

Glaxo Wellcome

Hoechst Marion Roussel

Merck Sharp \& Dohme

Novartis

Pfizer

Roche

Sanofi

Servier

\section{Grupos de Trabalho:}

\section{Grupo 1}

Diagnóstico e Classificação

Coordenador:

Décio Mion Jr (SP)

Altamiro Reis da Costa (RS)

Angela Pierin (SP)

Antonio Silveira Sbissa (SP)

Armando da Rocha Nogueira (RJ)

Ayrton Pires Brandão (RJ)

Edgard Pessoa de Mello (PE)

Fernando Nobre (SP)

Luiz Carlos Bodanese (RS)

Paulo Toscano (PA)

Sebastião Ferreira Filho (MG)

\section{Grupo 2}

Investigação Clínico-Laboratorial e

Decisão Terapêutica

Coordenador:

José Luiz Santello (SP)

Agostinho Tavares (SP)

José Márcio Ribeiro (MG)

Jorge Pinto Ribeiro (RS)

Juan Moreno Paz (PR)

Luiz Introcaso (DF)

Marco Antonio Gomes da Mota (AL)

Pedro Jabur (SP)

Raimundo Marques Nascimento (MG)

Rogério Andrade Mulinari (PR)

Rogério Baumgratz de Paula (MG) 


\section{Grupo 3}

\section{Abordagem Multiprofissional}

Coordenador:

Carlos Alberto Machado (SP)

Ana Luisa de Sousa (GO)

Clóvis Oliveira Andrade (SE)

Denise Mattos Dias (PE)

João Cabral Rocha

Lucélia Magalhães (BA)

Margarida Maria Verissimo Lopes (CE)

Marilda Lippi (SP)

Maria Fátima Azevedo (RN)

Nárcia Elisa Bellucci Kohlmann (SP)

Neide de Jesus (BA)

Paulo Cesar da Veiga Jardim (GO)

\section{Grupo 4}

\section{Tratamento Não-Medicamentoso}

Coordenador:

Hilton de Castro Chaves Jr. (SP)

Carlos Eduardo Negrão (SP)

Celso Amodeo (SP)

Cláudio Pereira da Cunha (PR)

Eliuden Galvão de Lima (ES)

Estelamaris Tronco Monego (GO)

Fátima Lúcia Machado Braga (PE)

Joel Heiman (SP)

José Gastão Rocha Carvalho (PR)

Natalino Salgado (MA)

Rui Póvoa (SP)

\section{Grupo 5}

\section{Tratamento Medicamentoso}

\section{Coordenador:}

Osvaldo Koblmann Jr. (SP)

Artur Beltrame Ribeiro (SP)

Daniel Rinaldi (SP)

Dante Marcelo Artigas Giogi (SP)

Emilton Lima Jr. (PR)

Fernando Antonio Almeida (SP)

Gilson Feitosa (BA)
Hélio Bernardes Silva (SP)

João Cesar Mendes Moreira (SP)

José Antonio Franchini Ramirez (SP)

Marcelo Marcondes Machado (SP)

Maria Helena Catelli Carvalho (SP)

Michel Batlouni (SP)

Oswaldo Luiz Ramos (SP)

Roberto Jorge da Silva Franco (SP)

Wille Oigman (RJ)

\section{Grupo 6}

\section{Tratamento da Hipertensão Arterial em}

Situações Especiais

Coordenador:

José Nery Praxedes (SP)

Alvaro Nagib Atallah (SP)

Andréa Brandão (RJ)

Istênio Paschoal (DF)

Ivan Cordovil (RJ)

Luiz A. H. Mariz (RJ)

Luiz Roberto Ramos (SP)

Maria Tereza Zanella (SP)

Maurício Wajngarten (SP)

Mauro A. Haidar (SP)

Vera Kock (SP)

\section{Grupo 7}

\section{Prevenção Primária}

Coordenador:

Armênio Costa Guimarães (BA)

Abrahão Afiune Neto (GO)

Ana Carolina Vasconcellos (PE)

Antonio Carlos Lopes (SP)

Airton Massaro (SP)

Cátia Palmeira (BA)

Eduardo Moacyr Krieger (SP)

Flávio Dani Fuchs (RS)

Jussara Melo (SP)

Marcus Vinícius Bolivar Malachias (MG)

Rafael Leite Luna (RJ)

Renan Alves da Cunha (DF) 